\title{
Discrimination of 16 Structural Isomers of Fucosyl Galactoside Based on Energy-Resolved Mass Spectrometry
}

\author{
Shusaku Daikoku, Takuro Ako, Rumiko Kato, Isao Ohtsuka, \\ and Osamu Kanie \\ Mitsubishi Kagaku Institute of Life Sciences (MITILS), Machida-shi, Tokyo, Japan
}

Glycans, a family of compounds often attached to proteins and ceramides, are diverse molecules involved in a wide range of biological functions. Their structural analysis is necessary and is often carried out at the microscale level. Methods based on mass spectrometry are therefore used, although they do not provide information regarding isomeric structures often found in glycan structures. If one finds "factors" characteristic of a certain isomer, this information can be used to elucidate an unknown oligosaccharide sequence. One potential technique is to use energy-resolved mass spectrometry (ERMS) that has been used to distinguish a pair of isomeric compounds. Thus, compounds in a combinatorial library might be effectively used for this purpose. We analyzed a set of 16 isomeric disaccharides, the structures of which consisted of all possible combinations of anomeric configurations and interglycosidic linkage positions. All of the compounds were distinguished based on ERMS where normal collision-induced dissociation could distinguish only seven compounds. Furthermore, it was shown that $\alpha$-glycosidic linkages of fucose were more reactive than the $\beta$-isomers and the secondary glycosides were more reactive than the primary glycosides. (J Am Soc Mass Spectrom 2007, 18, 1873-1879) (c) 2007 American Society for Mass Spectrometry

$\mathrm{M}$ ost oligosaccharides exist as a part of glycoproteins, proteoglycans, and glycolipids, and are involved in a wide range of important biological functions [1]. The structural characteristics of oligosaccharides are quite different from those of other biopolymers such as nucleic acids and peptides. The diversity is generated from sequential combination in the latter two types of polymers, whereas anomers, ring size, linkage position, branching, and sequence are factors in the case of oligosaccharides [2]. Furthermore, the regulatory mechanisms for oligosaccharides still need to be clarified and synthesis, which is not template dependent, thus creates further molecular diversity called glycoforms. Despite the difficulties encountered in structural determination, there are reports on how glycosylation as a type of posttranslational modification (PTM) of protein affects glycoprotein secretion [3, 4]. The involvement of PTM in cellular polarity has also been reported [5, 6]. Changes in composition of glycosphingolipids associated with development and differentiation are well recognized [8].

Considering the need for analyzing minute amounts of glycoconjugates obtained from biological samples

Address reprint requests to Dr. Osamu Kanie, Mitsubishi Kagaku Institute of Life Sciences (MITILS), 11 Minamiooya, Machida-shi, Tokyo 194-8511, Japan. E-mail: kokanee@mitils.jp and the existence of glycoforms, it is believed that the use of mass spectrometry-based analytical methods in the analysis of glycoconjugates is a logical consequence [8]. Structural analysis based on collision-induced dissociation (CID) is a very powerful technique in this regard [9], although it is not capable of elucidating isomeric compounds that are often found for oligosaccharides in general [10]. Despite this problem, it has been shown that there are spectral differences among isomeric compounds. In most cases, there are differences in signal intensities, whereas in others, different fragment ions can be found. Accumulation of the spectral data in a database facilitates identification of oligosaccharides based on spectral matching [11-17]. An important problem-the elucidation of totally unknown structures that are not present in any databasewill still remain after the introduction of this efficient methodology. In such a case, a general method for elucidating anomeric configurations and linkage positions is needed. Thus, a trend of research has emerged that addresses distinguishing and possibly finding the characteristics from a series of closely related oligosaccharides [11, 18, 19]. In 1988 a pioneering investigation was reported to address the energy dependence of linkage isomers based on collision-activated dissociation (CAD), where it was found that the lability of glycosidic bonds reflects the freedom of rotation of 
chemical bonds around a linkage for the dissociation [20]. Energy dependence of a pair of anomers was also addressed [21]. A series of reports suggested that the correlation of dissociation energy and the steric hindrance of a glycosidic linkage to be cleaved was shown to be applicable in real-world examples as well [22, 23]. On the other hand, an interesting approach to extract information contained in disaccharide structures was reported to analyze the relationship between the structures and mass spectral data based on multivariate analysis [24, 25]. However, the problem there was considered to be a lack of sufficient source materials. Our rationale was to determine the missing link between the above-cited approaches.

We have been attempting the extraction of an isomeric structure-oriented "parameter," based on energyresolved mass spectrometry (ERMS), and have reported that isomeric homooligomers and a series of synthetic compounds can be discriminated [26, 27]. Here in this report, we describe the results of ERMS analysis of a small combinatorial library [28, 29], consisting of a complete combination of compounds with a fucosyl galactose sequence and an important finding regarding anomeric structures.

\section{Experimental}

\section{Materials}

We synthesized all disaccharides used in the experiments [28, 29]. Synthesis was performed on a small scale where stereoselectivity was not controlled to obtain all anomers in one sequence of reactions. In this experiment, the octyl glycosides were used because they facilitate rapid isolation based on reverse-phase solid-phase extraction. Thus, the mixtures of compounds obtained were finally isolated using reversephase HPLC to attain pure individual compounds. Synthetic details and assignment of the compounds were previously reported [29].

\section{Instrumentation and Data Collection}

Samples of disaccharides were analyzed using a quadrupole ion trap mass spectrometer (QIT-MS) coupled with an electrospray interface (Bruker Esquire 3000 ${ }^{+}$, Bruker Daltonics GmbH, Bremen, Germany). Samples dissolved in $\mathrm{MeOH}(0.01-0.1 \mu \mathrm{mol} / \mathrm{ml})$ were introduced into the ion source by infusion (flow rate, 120 $\mu \mathrm{l} / \mathrm{h})$. The parameters for the analysis were: (1) "dry temperature": $250^{\circ} \mathrm{C}$, (2) nebulizer gas $\left(\mathrm{N}_{2}\right)$ : 10 psi, (3) dry gas $\left(\mathrm{N}_{2}\right)$ : $4.0 \mathrm{~L} / \mathrm{min},(4)$ "Smart frag.": off, (5) Scan range $\mathrm{m} / \mathrm{z}$ 50-750, (6) Compound stability: 300\%, (7) ICC target: 5000, (8) maximum acquisition time: $200 \mathrm{~ms}$, (9) average: 10 spectra, and (10) "cutoff": $27.6 \%(\mathrm{~m} / \mathrm{z}$ 127.0) of $m / z$ 461.2.

In our $\mathrm{MS}^{n}$ experiments, the end cap rf amplitude was raised by $0.02-\mathrm{V}$ increments until the precursor ion could no longer be detected (plateau at $<0.9 \%$ of total ion current). Only the end cap rf amplitude was con- trolled during the CID experiment. The He pressure was $4.86 \times 10^{-6} \mathrm{mbar}$ and the CID time was $40 \mathrm{~ms}$. Averages of $m-4$ spectra were used for CID experiments $(m=13-21$, where $m$ is the number of spectra obtained during the experiment); the first and the last two datasets, which are associated with a transient period to steady state, in a radio frequency (rf) amplitude step were not used to avoid any inaccuracy.

Isotopic peaks with $\left[\mathrm{I}^{i}+1\right]$ and $\left[\mathrm{I}^{i}+2\right]$, where $\mathrm{I}^{n}$ indicates a fragment ion, were included in the calculations (also see Data Manipulation). For the isolation of a product ion, $m / z \pm 2(w=2)$ were isolated and subjected to the CID experiments to include isotopes. Standard MS/MS spectra are the extracts of these ERMS at a designated amplitude.

\section{Data Manipulation}

To obtain graphs of the ERMS, the following equations


product ions- $\mathrm{I}^{1}, \mathrm{I}^{2}, \mathrm{I}^{3}, \ldots, \mathrm{I}^{i}$-the relative ion currents for individual ions were defined by

$$
{ }^{r e l} C=\frac{C^{I^{i}}}{C^{I^{p}}+\sum_{i=1}^{n} C^{I^{i}}} \times 100
$$

where ${ }^{r e l} \mathrm{C}$ indicates the ion current of a given ion among observed ions in percentage, $C^{I^{i}}$ is the observed ion current in focus, and $C^{I^{P}}$ is the ion current of a precursor ion. The calculations were performed using a program we developed with Excel (Excel 2000; Microsoft, Redlands, WA, USA), which was based on DSUM function and programmed to choose a range of isotopes $(w)$ to be taken into consideration $(w=2$ in the experiments).

\section{Sigmoidal Curve Fittings}

A set of $\mathrm{MS}^{n}$ data obtained at various rf amplitudes (end cap) on a mass spectrometer was analyzed using Excel where sets of peaks having certain $m / z$ values were treated as a series of data. The relative intensities over total ion current for the individual signals were obtained at each amplitude (Eq 1). The data were analyzed using Prism 4 software (GraphPad Software, San Diego, CA, USA). Individual data were fitted using the Boltzmann sigmoidal function with nonlinear regression analysis [Eq 2 (growth) and Eq 3 (decay)].

$$
\begin{aligned}
& y=\frac{a}{1+e^{[(b-x) c]}} \\
& y=\frac{a}{1+e^{[-(b-x) c]}}
\end{aligned}
$$

where the parameters $a, b$, and $c$, which indicate a maximum response, half value of the maximum re- 


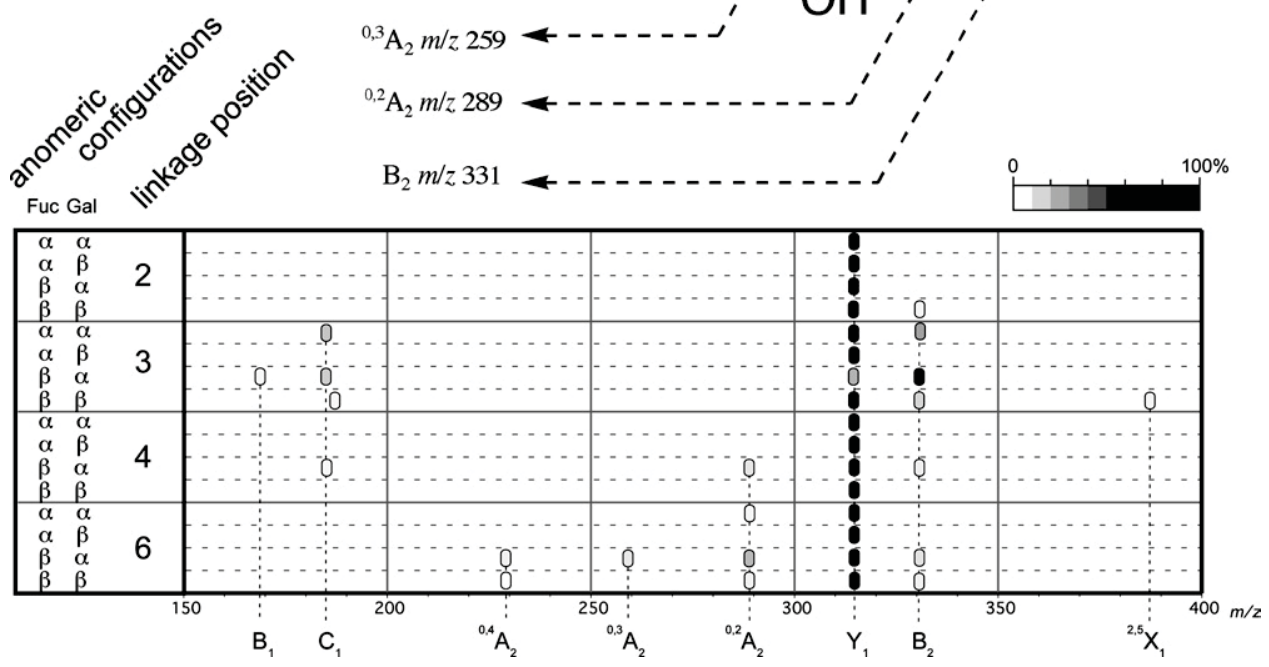

Figure 1. The fragmentation pattern of a series of disaccharides and the $\mathrm{MS}^{2}$ spectra show where the structures of $1 \rightarrow 6$ linked isomers were found only to describe the pattern. The peak intensities are indicated by the level of gray.

sponse, and a slope factor, respectively, were obtained for each curve.

In the series of data used in this report, the sigmoidal curves and the parameters were obtained by plotting regression curves by treating all the data obtained from the Excel program and plotting the regression curves.

\section{Results and Discussion}

\section{CID Analysis of All Compounds}

CID is very useful for extracting detailed structural information of a molecule in focus. The analysis is carried out at a certain CID energy, which is usually controlled by applying a certain voltage to end-cap electrodes, and results in a spectrum of fragment ions formed from a precursor ion [9]. Elucidation of molecular structure is carried out by analogy by solving a jigsaw puzzle and connecting fragments together. In such a case, information defined by an $m / z$ value is used; thus analysis of a particular precursor ion does not provide any information regarding isomeric structures. On the other hand, isomeric structures can often be distinguished when a pair of stereoisomers or linkage isomers is available. A series of naturally occurring oligosaccharides is not considered to contain sufficient information to extract any generalities regarding ano- meric configurations and linkage positions. In this regard, a series of compounds (16 isomers) that we have synthesized, consisting of all possible isomeric structures with a Fuc-Gal sequence, is considered to be a good candidate as the information source [28, 29]. We first analyzed these compounds by standard CID to see whether these closely related structures are distinguishable from one another.

All compounds were found as sodium adducts and were analyzed under CID conditions in which the precursor ions were completely attenuated. The resulting MS/MS spectra are shown in Figure 1 together with the fragmentation pattern. The relative labilities of fucosyl and galactosyl linkages are different, judging by the spectra, and the lability is greater for the cleavage of fucosyl glycosides. This is a commonly found phenomenon in low-energy CID analysis of oligosaccharides [30]. Thus, half of the compounds afforded $Y_{1}$ fragments associated with $\mathrm{B}_{1} / \mathrm{Y}_{1}$ cleavages only. It is obvious that such spectra do not provide any further information. On the other hand, all the compounds that afforded multiple fragment ions can be easily distinguished. Some of the characteristic features can be found as follows. In $2 \beta \beta$, ions associated with glycosyl cleavages for both Fuc and Gal were observed but no other ions were detected. For the spectra of $3 x \alpha$ and 
$3 \beta \alpha$, add $C_{1}$ ion as well as ions associated with Fuc- and Gal-cleavages. It is interesting that $B_{1}$ ion was observed only for the $\beta$-Fuc compound $(3 \beta \alpha)$, even though the difference between these two $(3 \alpha \alpha$ and $3 \beta \alpha)$ is the anomeric configurations of the Fuc unit only. This result is puzzling because both of the product ions, $B_{1}$ and $Y_{1}$ ions, share a similar structure; however, this may suggest that $\beta$ Fuc-Gal had more affinity with $\mathrm{Na}^{+}$compared with its counter $\alpha$-structure because a similar tendency was observed in a previous study [27]. In the CID spectrum of $3 \beta \beta,{ }^{2,5} \mathrm{X}_{1}$ ion and an unusual fragment ion with $\mathrm{m} / \mathrm{z} 187$ were observed as characteristic ions. The former ion is a result of Fuc ring rupture, which is rarely observed in low-energy CID of oligosaccharides, especially when located at a non-reducing end sugar. Compound $4 \beta \alpha$ produced ${ }^{0,2} \mathrm{~A}_{2}$ ion associated with rupture of $\mathrm{Gal}$ glycoside in addition to $\mathrm{Y}_{1}, \mathrm{~B}_{2}$, and $\mathrm{C}_{1}$ ions. Only $Y_{1}$ and ${ }^{0,2} A_{2}$ ions were observed for $6 \alpha x$. The spectra of $6 \beta \alpha$ and $6 \beta \beta$ were complex where ions that resulted from $G a l$ ring rupture such as $m / z 229\left({ }^{0,4} \mathrm{~A}_{2}\right)$, $m / z 259\left({ }^{0,3} \mathrm{~A}_{2}\right)$, and $m / z 289\left({ }^{0,2} \mathrm{~A}_{2}\right)$ were observed. Among these ions, the ${ }^{0,4} \mathrm{~A}_{2}$ ion contains Fuc with C5-C6 of Gal, and thus reflects the structure of a Fuc-6X linkage. The ${ }^{0,3} \mathrm{~A}_{2}$ ion was not observed for the latter compound.

It was observed that compounds closely related structurally consisting of anomers and linkage isomers could be distinguished by CID spectra when multiple ions were obtained. This indicates that the spectral differences often observed for isomeric natural oligosaccharides are not just a coincidence, and also supports the usefulness of methods based on spectral matching [11-17]. A problem, however, is that this is true only when a sufficient number of distinct signals were collected for the comparison. For example, in our case, half of the compounds were not distinguishable.

\section{ERMS Analysis}

Energy-resolved mass spectrometry (ERMS) has been used to investigate the structures of ions and noncovalently associated ions [31-40]. We have analyzed isomeric oligosaccharides based on ERMS and shown that (1) the primary glycosidic linkage is more stable than the secondary glycoside linkage; and (2) $\alpha$-glycosides are more labile than $\beta$-glycosides, through investigation of malto $(\alpha 1 \rightarrow 4)-$, isomalto $(\alpha 1 \rightarrow 6)-$, and cello $(\beta 1 \rightarrow 4)$-oligosaccharide [26] and trisaccharide libraries with the $\alpha / \beta$-Fuc- $(1 \rightarrow 6)-\alpha / \beta$-Gal- $(1 \rightarrow 6)-\alpha / \beta$-Glc-octyl sequence [27]. It is important to confirm the generality of these tendencies and at the same time to accumulate such data regarding the structure-related characteristics for future development of methods to identify novel glycoconjugates. To attain these objectives, we analyzed the ERMS data of the series of Fuc-Gal disaccharides.

ERMS obtained for 16 sodiated isomeric compounds are presented in Figure 2. It is evident that product ion(s) were gradually formed as the signal intensity of parent ions decreased with increased voltage for the supplementary dipolar rf applied on end-cap electrodes. The data shown earlier are the intensities of product ions obtained at a voltage where the individual precursor ions reached zero intensity. As indicated in the ERMS of the isomeric compounds, these spectra provide information related to the energy required for the dissociation and generation of ions in addition to information on the mass-to-charge ratios. We were interested to determine whether we could find any further differences from the standard MS/MS shown earlier, and whether we could differentiate previously undistinguishable compounds. Furthermore, we hoped that we might be able to extract information regarding anomeric configurations and/or linkage positions. For this purpose, the decay curves of precursor ions were selected and plotted together according either to each anomeric configuration (shown on right-hand side for $\alpha / \alpha, \alpha / \beta, \beta / \alpha$, and $\beta / \beta$ from top to bottom) or to each linkage position (shown at bottom for $1 \rightarrow 2,1 \rightarrow 3,1 \rightarrow 4$, and $1 \rightarrow 6$ from left to right).

At first, we focused on the ERMS of those that resulted in the production of a single ion $(\mathrm{m} / \mathrm{z} 315)$ in standard CID experiments, that is, spectra for $2 \alpha \alpha, 2 \alpha \beta$, $2 \beta \alpha, 3 \alpha \beta, 4 \alpha \beta, 4 \beta \beta$, and $6 \alpha \beta$. It seemed that some could be distinguished by visual inspection. For example, the decay curve of the precursor and generation curve for the product ion for each ERMS cross at different amplitudes of end-cap voltage in $2 \alpha \alpha, 2 \alpha \beta$, and $2 \beta \alpha$, where the apparent order was $2 \alpha \beta<2 \alpha \alpha<2 \beta \alpha$. This suggests that ERMS can be used for the identification of oligosaccharides more effectively than standard MS/MS spectra. Although it is possible to discuss the differences in all the spectra where only a single ion was observed in the same manner, we will stop here and discuss it later.

To look into the spectral differences in a more systematic manner, let us analyze each linkage series. For this type of comparison to be made for any ERMS regardless of the number of product ions, only decay curves were used. Because the generation of individual fragment ions is related to the activation energy of the corresponding precursor ions, it may be possible to find correlation between the curve and subtle differences in the structures under comparison. The individual series of graphs (bottom left four of Figure 2) for each linkage series are quite dramatic, where it was shown that ions with an $\alpha$-Fuc residue tended to dissociate at lower energy in all cases. It was observed that the dissociation curves of compounds with $\alpha / \beta$ - and $\alpha / \alpha$-configurations were reversed in $1 \rightarrow 3$ linked disaccharides. The reactivity of a series of compounds is dependent on an anomeric configuration regardless of the linkage position, and the order is $\alpha / \beta \sim$ $\alpha / \alpha<\beta / \beta<\beta / \alpha$ (Fuc/Gal). The observed tendency coincides with previous results obtained for the fucosyl and galactosyl cleavages in a series of trisaccharides. Thus, it is considered that the phenomenon is a general event in cases in which the orientations of $2-\mathrm{OH}$ and anomeric configuration are fixed. Furthermore, it was found that $\alpha$-glycosides (1,2-cis-axial) were more labile than $\beta$-glyco- 


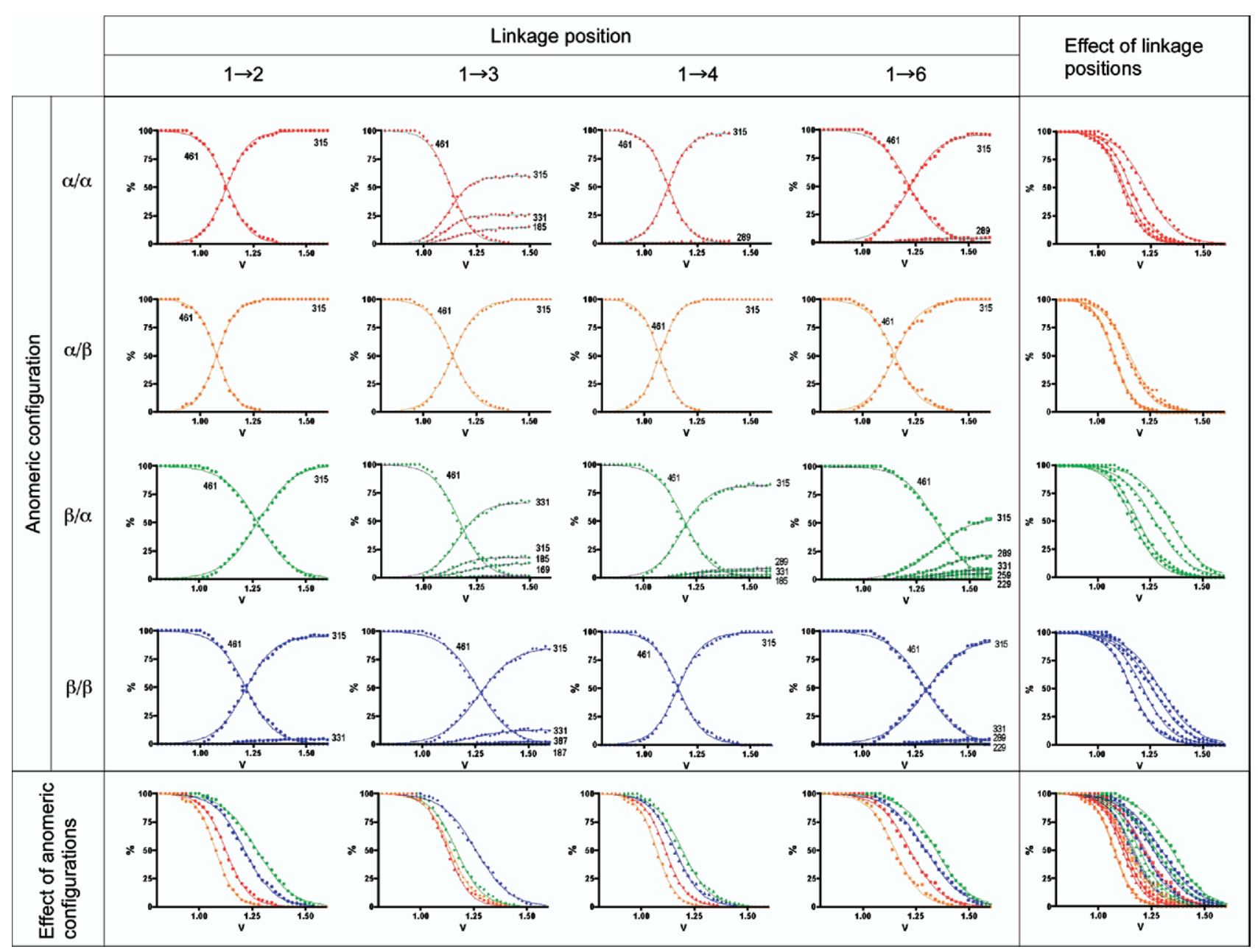

Figure 2. The ERMS of a set of compounds consisted of all combinations of anomeric configurations and glycosidic linkage positions. Individual plots were subjected to nonlinear regression analysis using the Bolzmann function [Eq 2 (growth), Eq 3 (decay)].

sides (1,2-trans-equatorial). Generally it is accepted that $\alpha$-anomers are thermodynamically more stable than $\beta$-anomers [41-43], which is inconsistent with our observation in the low-energy CID process. Extensive theoretical calculations are under way to explain the phenomena.

Next, we focused on the anomer series (top four graphs on right side of Figure 2). The order of lability of linkage isomers in each anomer series were $4 \approx 2<3<$ $6,4 \approx 2<3 \approx 6,3<4<2<6$, and $4<2<3<6$, for $\alpha / \alpha, \alpha / \beta, \beta / \alpha$, and $\beta / \beta$ (Fuc/Gal), respectively. The order was not identical but it is obvious that secondary glycosides of Fuc-Gal were more reactive than primary (6-linked) disaccharides. These results again supported the above-described phenomena observed previously; however, it is not easy to explain in connection with the well-accepted reactivity of glycosidic linkages. Similar phenomena have been reported as well [20-23, 26, 27], which suggests this is a common feature in the CID process and could be explained by the diffusion of given kinetic energy and further higher conversion into heat in primary glycosides because of the flexibility and existence of more rotamers in the structure [20].
A graph containing all of the preceding decay curves is shown at the bottom right corner of Figure 2. One of our initial aims was to distinguish these isomers from each other. As is obvious from the graph, all 16 isomeric structures can be distinguished, although the adjacent energy-resolved curves (ERCs) are quite close to each other. Among them, 2- and 4-linked and 3- and 6-linked compounds in $\alpha / \beta$ (Fuc/Gal) series are difficult to distinguish. These compounds each gave rise to a single product ion. Thus, distinguishing them is still difficult based on visual inspection of ERMS. As a result, three of seven compounds that resulted in the formation of a single product ion were further distinguished according to ERMS analysis.

\section{Scatterplot Analysis of Parameters Obtained from ERCs}

As explained earlier, discrimination can still be difficult despite the power of ERMS. To address this issue, we further examined the scatterplot analysis of two param- 


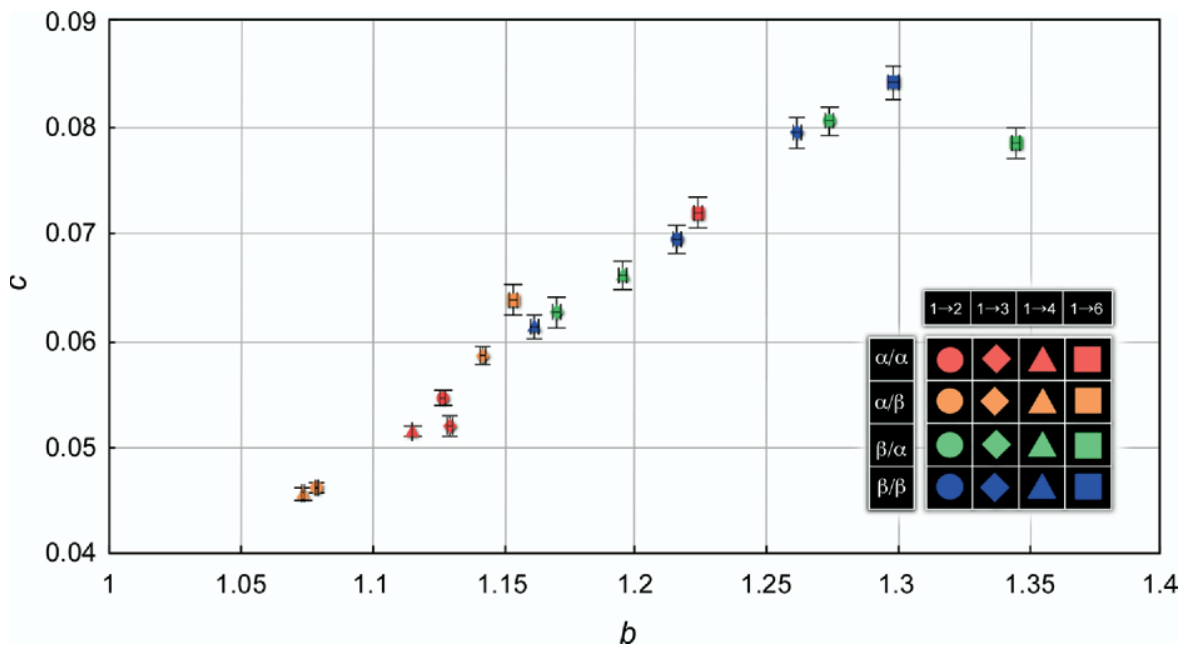

Figure 3. A scatterplot of two parameters, $b$ and $c$, obtained from Eq 3. Each structure in a plot was coordinated by colors and symbols as shown. Standard errors obtained by a non-linear regression analysis of each sigmoidal decay curve are shown.

eters ( $b$ and $c$; see Experimental section) obtained for individual ERCs after nonlinear regression analysis of the decay curves using the Boltzmann sigmoidal function (Figure 3). Unlike visual inspection carried out earlier, this scatterplot analysis can be made quantitatively. According to the plot shown in Figure 3, the parameters obtained for the dissociation for all compounds were resolved. Let us focus on problematic ERCs found previously, that is, 2- and 4-linked compounds in the $\alpha / \beta$ (Fuc/Gal) series. These parameters were rather close to each other, although it might be possible to distinguish them based on the $b$ values where $b=1.078, \mathrm{SE}=0.00049$ and $b=1.073, \mathrm{SE}=$ 0.00067 for $\alpha$-Fuc- $(1 \rightarrow 2)-\beta$-Gal and $\alpha$-Fuc- $(1 \rightarrow 4)-\beta$-Gal, respectively. The parameters for the 3- and 6-linked compounds in the $\alpha / \beta$ (Fuc/Gal) series were $b=1.141$, $\mathrm{SE}=0.00100$ and $b=1.153, \mathrm{SE}=0.00167$, and $c=$ $0.0586, \mathrm{SE}=0.00088$ and for $c=0.0637, \mathrm{SE}=0.00147$ for $\alpha$-Fuc- $(1 \rightarrow 3)-\beta$-Gal and $\alpha$-Fuc- $(1 \rightarrow 6)-\beta$-Gal, respectively. Thus, it was possible to distinguish structurally related compounds, which could not be distinguished by other methods. Also, it is clear from the scatterplot that all 16 related compounds have distinguishable dissociation energies. There seems to exist a correlation between $b$ and $c$ values, but we do not have a conclusive explanation for this. Regardless of an emerging issue, it was shown that the scatterplot analysis of ERMS was a powerful technique to distinguish isomeric compounds. This may be effectively used in discriminating compounds or ions generated under CID conditions.

\section{Conclusion}

The diverse glycans form even more complex glycoconjugates such as glycoproteins, proteoglycans, and glycolipids in living systems. For the analysis of these structures, mass spectrometry-based methods are necessary. The diversity of glycan structure is created by the existence of anomers, linkage isomers, and branching. Although it is known there are differences in fragment patterns among isomeric structures, the discrimination and identification depend on a standard compound whose structure is known. The use of a "known compound" as a reference material is problematic in the analysis of a "novel glycan" because of the lack of a reference. To overcome this problem, fundamental research is vital to understand the general characteristics hidden inside the glycan structure. Therefore, we carried out the ERMS analysis of a small library of compounds as an information source. As a result, it was found that analysis of ERCs of the precursor ions alone surpasses the analysis based on traditional MS/MS. It is believed this is especially useful for structural identification methods that refer to a database. ERMS also contains $\mathrm{m} / \mathrm{z}$ values and signal intensity, and thus provides extremely rich structural information that enabled the discrimination of 16 isomeric compounds. Accumulation of this type of information may lead to the development of a new method for the structural elucidation of glycoconjugates. We propose obtaining curve parameters of ERMS from various compounds recorded on different mass spectrometers, which may be very useful in finding generalities and differences of data. We hope that these data will be used to find a way to correct data obtained from different spectrometers. With such a method, the CID data can be interconverted to be compared.[7]

\section{Acknowledgments}

The authors thank Sachiko Mutsuga for her excellent technical assistance. This research was supported by the Key Technology Research Promotion Program of the New Energy and Industrial Technology Development Organization (NEDO), which is under the auspices of the Ministry of Economy, Trade and Industry (METI) of Japan, and by Mitsubishi Chemical Corporation. 


\section{References}

1. Varki, A. Biological Roles of Oligosaccharides: All of the Theories Are Correct. Glycobiology 1993, 3, 97-130.

2. Laine, R. A. Invited Commentary: A Calculation of All Possible Oligosaccharide Isomers Both Branched and Linear Yields $1.05 \times 10^{12}$ Structures for a Reducing Hexasaccaride: The Isomer Barrier to Development of Single-Method Saccharide Sequencing or Synthesis Systems. Glycobiology 1994, 4, 759-767.

3. Dubé, S.; Fisher, J. W.; Poewll, J. S. Glycosylation at Specific Sites of Erythropoietin Is Essential for Biosynthesis, Secretion, and Biological Function. J. Biol. Chem. 1988, 263, 17516-17521.

4. Roth, J. Protein N-Glycosylation along the Secretory Pathway: Relationship to Organelle Topography and Function, Protein Quality Control, and Cell Interactions. Chem. Rev. 2002, 102, 285-303.

5. Rodriguez-Boulan, E.; Gonzalez, A. Glycans in Post-Golgi Apical Targeting: Sorting Signals or Structural Props? Trends Cell Biol. 1999, 9, 291-294

6. Benting, J. H.; Rietveld, A. G.; Simons, K. N-Glycans Mediate the Apical Sorting of a GPI-Anchored, Raft-Associated Protein in Madin-Darby Canine Kidney Cells. J. Cell Biol. 1999, 146, 313-320.

7. Yamashita, T.; Wada, R.; Sasaki, T.; Deng, C.; Bierfreund, U.; Sandhoff, K.; Proia, R. L. A Vital Role for Glycosphingolipid Synthesis during Development and Differentiation. Proc. Natl. Acad. Sci. U.S.A. 1999, 96, 9142-9147.

8. Chaplin, M. F.; Kennedy, J.F., Eds. Carbohydrate Analysis. Oxford, UK: IRL Press; 1994

9. Hayes, R. N.; Gross, M. L. Collision-Induced Dissociation. In Methods in Enzymology: Mass Spectrometry, Vol. 193, McCloskey, J. A., Ed.; Academic Press: San Diego, CA, 1990, pp 237-263.

10. Dell, A. FAB-Mass Spectrometry of Carbohydrates. Adv. Carbohydr. Chem. Biochem. 1987, 45, 19-72.

11. Mulroney, B.; Traeger, J. C.; Stone, B. A. Determination of Both Linkage Position and Anomeric Configuration in Underivatized Glucopyranosyl Disaccharides by Electrospray Mass Spectrometry. J. Mass Spectrom. 1995, 30, 1277-1283.

12. Viseux, N.; de Hoffmann, E.; Domon, B. Structural Assignment of Permethylated Oligosaccharide Subunits Using Sequential Tandem Mass Spectrometry. Anal. Chem. 1998, 70, 4951-4959.

13. Takegawa, Y.; Deguchi, K.; Ito, S.; Yoshioka, S.; Sano, A.; Yoshinari, K.; Kobayashi, K.; Nakagawa, H.; Monde, K.; Nishimura, S.-I. Assignment and Quantification of 2-Aminopyridine Derivatized Oligosaccharide Isomers Coeluted on Reversed-Phase HPLC/MS by $\mathrm{MS}^{n}$ Spectral Library. Anal. Chem. 2004, 76, 7294-7303.

14. Kameyama, A.; Kikuchi, N.; Nakaya, S.; Ito, H.; Sato, T.; Shikanai, T.; Takahashi, Y.; Takahashi, K.; Narimatsu, H. A Strategy for Identification of Oligosaccharide Structures Using Observational Multistage Mass Spectral Library. Anal. Chem. 2005, 77, 4719-4725.

15. Ashline, D.; Singh, S.; Hanneman, A.; Reinhold, V. Congruent Strategies for Carbohydrate Sequencing. 1. Mining Structural Details by MS $^{n}$. Anal. Chem. 2005, 77, 6250-6262.

16. Zhang, H.; Singh, S; Reinhold, V. Congruent Strategies for Carbohydrate Sequencing. 2. FragLib: An $\mathrm{MS}^{n}$ Spectral Library. Anal. Chem. 2005, 77, 6263-6270

17. Lapadula, A. J.; Hatcher, P. J.; Hanneman, A. J.; Ashline, D. J.; Zhang, H.; Reinhold, V. Congruent Strategies for Carbohydrate Sequencing. 3. OSCAR: An Algorithm for Assigning Oligosaccharide Topology from MS $^{n}$ Data. Anal. Chem. 2005, 77, 6271-6279.

18. Dallinga, J. W.; Heerma, W. Positive Ion Fast AtomBombardment Mass Spectrometry of Some Small Oligosaccharides. Biol. Mass Spectrom. 1991, 20, 215-231.

19. Blok-Tip, L.; van der Kert-van Hoof, A.; Heerma, W.; Haverklamp, J.; Kovácik, V.; Hirsch, J. Determination of Linkage Positions in Peracetylated (Methyl) Xylo-oligosaccharides with Fast Atom Bombardment Mass Spectrometry. Biol. Mass Spectrom. 1993, 22, 474-480.

20. Laine, R. A.; Pamidimukkala, K. M. French, A. D. Hall, R. W. Abbas, S. A.; Jain, R. K.; Matta, K. L. Linkage Position in Oligosaccharides by Fast Atom Bombardment Ionization, Collision-Activated Dissociation, Tandem Mass Spectrometry and Molecular Modeling. L-Fucosyl $-(\alpha 1 \rightarrow \mathrm{X})-\mathrm{D}-\mathrm{N}$-acetyl-D-glucosaminyl $-(\beta 1 \rightarrow 3)-\mathrm{D}$ galactosyl $\mathrm{p}_{\mathrm{p}}-(\beta 1-\mathrm{O}-\mathrm{methyl})$ Where $\mathrm{X}=3,4$, or 6 . J. Am. Chem. Soc. 1988, 110, 6931-6939.

21. Mendonca, S.; Cole, R. B.; Zhu, J.; Cai, Y.; French, A. D.; Johnson, G. P.; Laine, R. A. Incremented Alkyl Derivatives Enhance Collision Induced Glycosidic Bond Cleavage in Mass Spectrometry of Disaccharides. J. Am. Soc. Mass Spectrom. 2003, 14, 63-78.
22. Laine, R. A.; Yoon, E.; Mahier, T. J.; Abbas, S.; de Lappe, B.; Jain R.; Matta, K. Non-reducing Terminal Linkage Position Determination in Intact and Permethylated Synthetic Oligosaccharides Having a Penultimate Amino Sugar: Fast Atom Bombardment Ionization, CollisionInduced Dissociation and Tandem Mass Spectrometry. Biol. Mass Spectrom. 1991, 20, 505-514.

23. Xue, J.; Song, L.; Khaja, S. D.; Locke, R. D.; West, C. M.; Laine, R. A Matta, K. L. Determination of Linkage Position and Anomeric Configuration in Hex-Fuc Disaccharides Using Electrospray Ionization Tandem Mass Spectrometry. Rapid Commun. Mass Spectrom. 2004, 18, 1947-1955.

24. Fängmark, I.; Jansson, A.; Nilsson, B. Determination of Linkage Position and Anomeric Configuration in Glucose-Containing Disaccharide Alditols by Multivariate Analysis of Data from Mass Spectrometry. Anal. Chem. 1999, 71, 1105-1110.

25. Higgins, M. K.; Bly, R. S.; Morgan, S. L. Differentiation of Isomeric Alditol Hexaacetates and Identification of Aldohexoses by Electron Impact Mass Spectrometry. Anal. Chem. 1994, 66, 2656-2668.

26. Kurimoto, A.; Daikoku, S.; Mutsuga, S.; Kanie, O. Analysis of EnergyResolved Mass Spectra at MS $^{n}$ in a Pursuit to Characterize Structural Isomers of Oligosaccharides. Anal. Chem. 2006, 78, 3461-3466.

27. Daikoku, S. Ako, T.; Kurimoto, A.; Kanie, O. Anomeric Information Obtained from a Series of Synthetic Trisaccharides Using EnergyResolved Mass Spectra. J. Mass Spectrom. 2007, 42, 714-723.

28. Kanie, O.; Ohtsuka, I.; Ako, T.; Daikoku, S.; Kanie, Y.; Kato, R Orthogonal Glycosylation Reactions on Solid-Phase and the Synthesis of a Library Consisting of a Complete Set of Fucosyl Galactose Isomers. Angew. Chem. Int. Ed. 2006, 45, 3851-3854.

29. Ohtsuka, I.; Ako, T.; Kato, R.; Daikoku, S.; Koroghi, S.; Kanemitsu, T.; Kanie, O. Synthesis of a Fucosyl Galactose Library Consisting of a Complete Set of Anomeric Configurations and Linkage Positions. Carbohydr. Res. 2006, 341, 1476-1487.

30. Harvey, D. J.; Mattu, T. S.; Wormald, M. R.; Royle, L.; Dwek, R. A.; Rudd, P. M. "Internal Residue Loss": Rearrangements Occurring during the Fragmentation of Carbohydrates Derivatized at the Reducing Terminus. Anal. Chem. 2002, 74, 734-740.

31. McLuckey, S. A.; Glish, G. L.; Cooks, R. G. Kinetic Energy Effects in Mass Spectrometry/Mass Spectrometry using a Sector/Quadrupole Tandem Instrument. Int. J. Mass Spectrom. Ion. Phys. 1981, 39, 219-230.

32. Fetterolf, D. D.; Yost, R. A. Energy-Resolved Collision-Induced Dissociation in Tandem Mass Spectrometry. Int. J. Mass Spectrom. Ion. Phys. 1982, 44, 37-50.

33. Verma, S.; Ciupek, J. D.; Cooks, R. G. Isomeric Structure Distinction Using Energy and Angular Resolution: $\mathrm{C}_{3} \mathrm{H}_{6} \mathrm{O}^{+}$. Int. J. Mass Spectrom. Ion. Proc. 1984, 62, 219-225.

34. Bursey, M. M.; Nystrom, J. A. Survey of Reaction Types in Low-Energy Collisional Activation of Protonated Methyl Alkyl Ketones. Anal. Chem. Acta 1984, 159, 265-274.

35. Williams, J. D.; Cox, K. A.; Cooks, R. G.; McLuckey, S. A.; Hart, K. J.; Goeringer, D. E. Resonance Ejection Ion Trap Mass Spectrometry and Nonlinear Field Contributions: The Effect of Scan Direction on Mass Resolution. Anal. Chem. 1994, 66, 725-729.

36. Hart, K. J.; McLuckey, S. A. Relative Dissociation Energy Measurements Using Ion Trap Collisional Activation. J. Am. Soc. Mass Spectrom. 1994, 5 , 250-259.

37. Favretto, D.; Guidugli, F.; Seraglia, R.; Traldi, P. Ursini, F.; Sevanian, A. Collisional Spectroscopy Approaches in the Characterization of 7-alphaand 7-beta-Hydroxycholesterol. Rapid Commun. Mass Spectrom. 1991, 5, $240-244$.

38. Gabelica, V.; Galic, N.; De Pauw, E. On the Specificity of Cyclodextrin Complexes Detected by Electrospray Mass Spectrometry. J. Am. Soc. Mass Spectrom. 2002, 13, 946-953.

39. Crowe, M. C.; Brodbelt, J. S. Evaluation of Noncovalent Interactions between Peptides and Polyether Compounds via Energy-Variable Collisionally Activated Dissociation. J. Am. Soc. Mass Spectrom. 2003, 14, $1148-1157$.

40. Broeren, M. A. C.; van Dongen, J. L. J.; Pittelkow, M.; Christensen, J. B. van Genderen, M. H. P.; Meijer, E. W. Multivalency in the Gas Phase: The Study of Dendritic Aggregates by Mass Spectrometry. Angew. Chem. Int. Ed. 2004, 43, 3557-3562.

41. Edward, J. T. Stability of Glycosides to Acid Hydrolysis. Chem. Ind. 1955, 1102-1104.

42. Lemieux, R. U. Effects of Unshared Pairs of Electrons and Their Solvation on Conformational Equilibria. Pure Appl. Chem. 1971, 25, 527-548.

43. Kirby, A. Stereoelectronic Effects on Acetal Hydrolysis. Acc. Chem. Res. 1984, 17, 305-311. 